Monatshefte für Chemie 107, 439-447 (1976)

( ) by Springer-Verlag 1976

\title{
Sila-Analoga des Mebrophenhydramins
}

\author{
Sila-Pharmaka, 3. Mitt. ${ }^{1}$ \\ Von \\ Reinhold Tacke ${ }^{2}$ und Ulrich Wannagat \\ Institut für Anorganische Chemie, Technische Universität Braunschweig, \\ Bundesrepublik Deutschland
}

(Eingegangen am 24. September 1975)

\section{Sila-Analogues of Mebrophenhydramine}

Sila-analogues A 2, B 2 and C 2 of the drug mebrophenhydramine from the class of benzhydryl ethers were synthesized for the first time by the steps shown in scheme 1 , and they and their precursors I-III were characterized by their physical (Table 1) and chemical properties, and their structures confirmed by NMR, mass and infrared spectroscopy (Tables 3-5). The histaminolytic and anticholinergic effects of A 2 and $\mathbf{C} 2$ were investigated and compared with some structure-activity relationships of analogue carbon compounds.

\section{Einführung}

Vergleichende pharmakologische Untersuchungen ${ }^{3}$ der Antihistaminika Mebrophenhydramin $(E l=\mathrm{C}, X=\mathrm{Br})$ und Mephenhydramin ( $E l=\mathrm{C}, X=\mathrm{H}$ ) haben ergeben, daß die brom-substituierte Verbindung bei verschiedenen Testmethoden und Applikationsarten eine bedeutend größere Antihistamin-Wirkung besitzt. Außerdem tritt diese Wirkung rascher ein und ist weitaus protrahierter. Untersuchun. gen der akuten Toxizität an Māusen zeigten, daß das Mebrophen. hydramin nur halb so giftig ist wie das Mephenhydramin, so daß die brom-substituierte Verbindung insgesamt den besseren therapeutischen Index aufweist ${ }^{3}$.

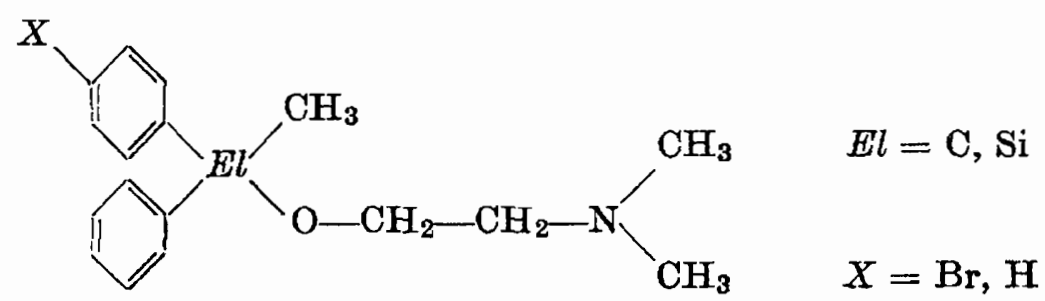


In Fortführung unserer Untersuchungen über Sila-Pharmaka stellten wir weitere Silicium-Analoga der Antihistaminika des ColaminTyps (vgl. 2. Mitt. ${ }^{1}$ ) her. In früheren Mitteilungen hatten wir bereits über die Darstellung und Eigenschaften eines Sila-Mephenhydramins ${ }^{4}$, Sila-Chlorphenoxamins ${ }^{1}$ und Sila-Clofenetamins ${ }^{1}$ berichtet. Es sollen nun die Ergebnisse der Untersuchungen über ein Sila-Mebrophenhydramin ("Si-Membryl", $E l=\mathrm{Si}, X=\mathrm{Br}$ ) und dessen Homologe (Variationen der Aminogruppe) mitgeteilt werden.

\section{Zur Darstellung des Sila-Mebrophenhydramins sowie seiner Homologen $\mathbf{B} 2$ und $\mathbf{G} 2$}

Die Benzhydryläther Mebrophenhydramin (A 1) und das Homologe B 1 wurden, von p-Brombenzophenon ausgehend, durch Umsetzung mit Methylmagnesiumjodid, Metallierung mit Natriumamid und NaClEliminierung mit 1-Chlor-2-dialkylaminoäthan dargestellt ${ }^{5}$ (Skizze 1).

Die Synthese des Sila-Mebrophenhydramins erfolgte auf zwei verschiedenen Wegen:

1. Umsetzung des Methyltrichlorsilans mit Phenylmagnesiumbromid führt über Rk. (1) zum bereits bekannten Methyl-phenyldichlorsilan (Darstellung und Eigenschaften sind in 4 beschrieben), das sich durch erneute Umsetzung mit p-Bromphenylmagnesiumbromid (Rk. 2) in das p-Bromphenyl-methyl-phenyl-chlorsilan (I) überführen läßt. Dieses reagiert mit 2-Dimethylaminoäthanol (Rk. 3) in guter Ausbeute zu A 2.

Analog wurden B 2 und C 2 durch Umsetzung von I mit 2-Diäthylaminoäthanol bzw. 2-Morpholinoäthanol dargestellt.

2. p-Bromphenyl-(methyl)-phenyl-(hydrido)silan (III) wurde, von Dichlor-(methyl)-(hydrido)silan ausgehend, durch stufenweise Umsetzung (Rkk. 5-7) mit Phenyl- und p-Bromphenylmagnesiumbromid über das p-Bromphenyl-chlor-(methyl)-(hydrido)silan (II) bzw. Chlor-(methyl)-phenyl-(hydrido)silan (beschrieben in ${ }^{4}$ ) dargestellt. Die weitere Umsetzung von III mit 2-Dimethylaminoäthanol in Gegenwart katalytischer Mengen des entsprechenden Lithiumalkoxids führt unter. Wasserstoffentwicklung in nahezu quantitativer Ausbeute zu A 2.

\section{Experimenteller Teil}

Die Darstellung von I-III sowie A 2-G 2 erfolgte in der gleichen Woise wie bei den in ${ }^{1}$ beschriebenen analogen p-chlor-substituierten Verbindungen. Für experimentelle Details sei auf die entsprechenden Arbeitsvorschriften in ${ }^{1}$ verwiesen. 
Skizze 1. Zur Darstellung des Mebrophenhydroamins (A 1), des Homologen B 1 sowie der Sila-Analoga A 2 - C 2<smiles>C[Si](Cl)(Cl)Cl</smiles><smiles>C[As](Cl)(c1ccccc1)c1ccc(Br)cc1</smiles>

(1) $\underline{A} 2, \underline{B} 2, \underline{C} 2$

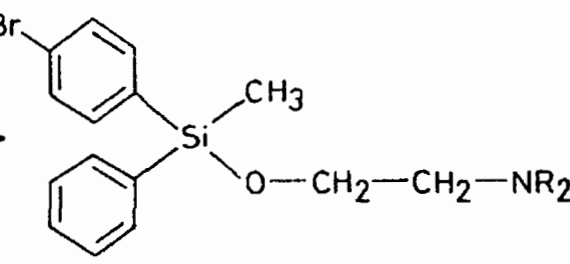

\begin{tabular}{c|ccc}
$\mathrm{NR}_{2}$ & $\frac{\mathrm{N}\left(\mathrm{CH}_{3}\right)_{2}}{\mathrm{~N}\left(\mathrm{C}_{2} \mathrm{H}_{5}\right)_{2}}$ & $\underline{\mathrm{B}}^{2}$ & $\underline{\mathrm{C}}{ }^{2}$
\end{tabular}
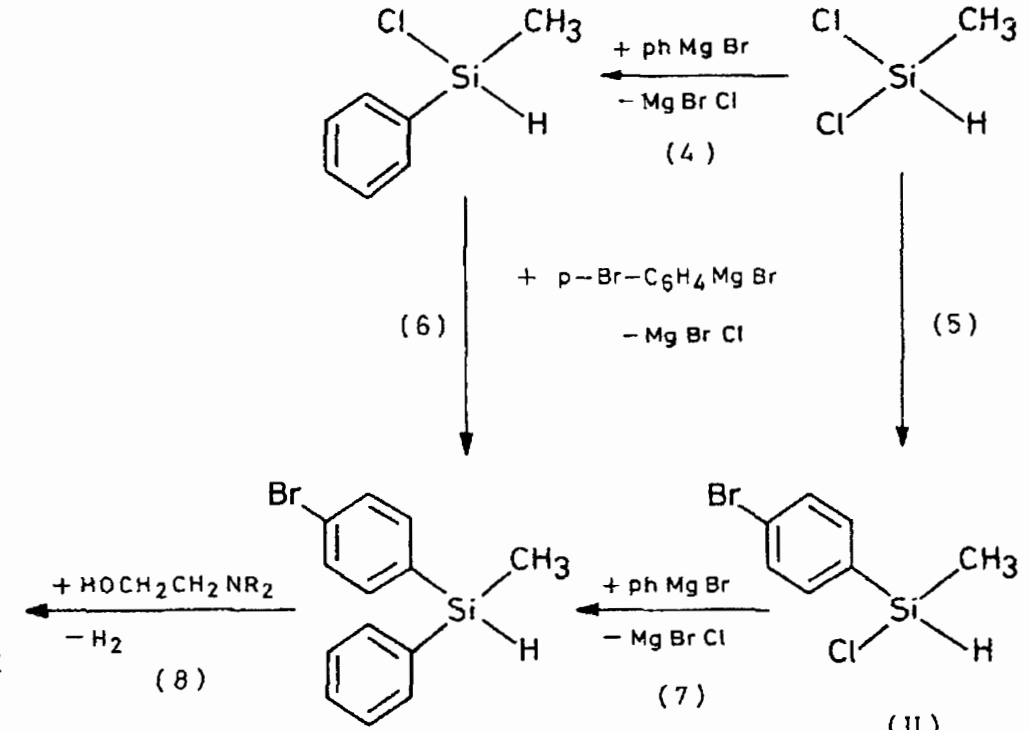

A 2

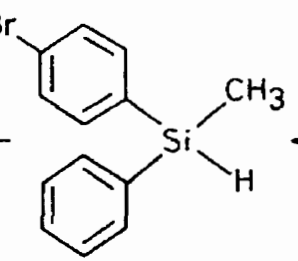

(III)

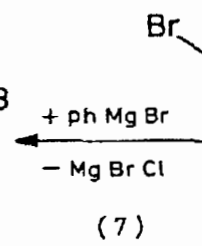

(7)

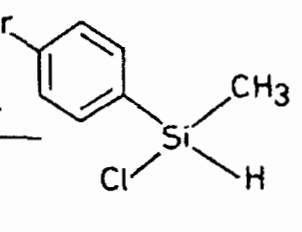

(II)

$\mathrm{ph}=\mathrm{C}_{6} \mathrm{H}_{5}$

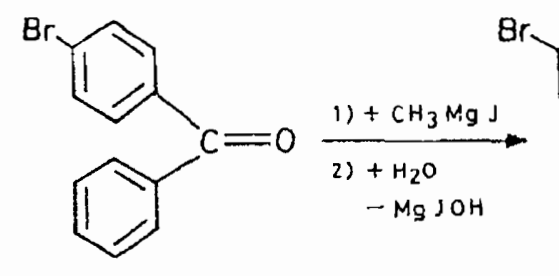<smiles>CC(O)(c1ccccc1)c1ccc(I)cc1</smiles>

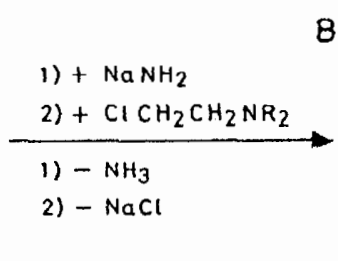<smiles>CC(OCC[N+](=O)[O-])(c1ccccc1)c1ccc(Br)cc1</smiles> 
p-Bromphenyl-methyl-phenyl-chlorsilan (I): Gemäß Rk. (2) durch Zutropfen einer aus 29,2 g (1,2 Mol) $\mathrm{Mg}$ und 283,1 g (1,2 Mol) 1,4-Dibrombenzol in $900 \mathrm{ml}$ Diäthyläther $(=\ddot{A}$ ) bereiteten Grignardlösung zu $200,7 \mathrm{~g}$ (1,05 Mol) Methyl-phenyl-dichlorsilan in $500 \mathrm{ml} \ddot{A}$. Ausb. $95 \mathrm{~g}(29 \%)$.

p-Bromphenyl-methyl-chlor-(hydrido)silan (II): Gemäß Rk. (5) durch Zutropfen einer aus $12,4 \mathrm{~g}(0,51 \mathrm{Mol}) \mathrm{Mg}$ und $118 \mathrm{~g}(0,5 \mathrm{Mol})$ 1,4-Dibrombenzol in $450 \mathrm{ml} \ddot{A}$ bereiteten Grignardlösung zu 57,5 g (0,5 Mol) Methyldichlor-(hydrido)silan in $250 \mathrm{mI} \ddot{A}$. Ausb. $30,6 \mathrm{~g} \mathrm{(26 \% ).}$

p-Bromphenyl-methyl-phenyl-(hydrido)silan (III): a) Gemäß Rk. (7) duroh Zutropfen von $117,8 \mathrm{~g}(0,5 \mathrm{Mol})$ II in $100 \mathrm{ml} \ddot{A}$ zu einer aus $12,4 \mathrm{~g}$ $(0,51 \mathrm{Mol}) \mathrm{Mg}$ und $78,5 \mathrm{~g}(0,5 \mathrm{Mol})$ Brombenzol in $450 \mathrm{ml} \ddot{A}$ bereiteten Grignardlösung. Ausb. $117,8 \mathrm{~g}(85 \%)$.

b) Gemäß Rk. (6) durch Zutropfen von 78,3 g (0,5 Mol) Methyl-phenylchlor-(hydrido)silan in $100 \mathrm{ml} \ddot{A}$ zu einer aus $12,4 \mathrm{~g}(0,51 \mathrm{Mol}) \mathrm{Mg}$ und $118 \mathrm{~g}(0,5 \mathrm{Mol})$ 1,4-Dibrombenzol in $450 \mathrm{ml} \ddot{A}$ bereiteten Grignardlösung. Ausb. 40,2 g (29\%).

$N, N$-Dimethyl-2-(p-bromphenyl-methyl-phenylsilyloxy)-äthylamin (SilaMebrophenhydramin, A 2): a) Gemäß Rk. (3) durch Zutropfen von $31,2 \mathrm{~g}$ $(0,1 \mathrm{Mol}) \mathrm{I}$ in $150 \mathrm{mI}$ Petroläther $\left(40 / 60^{\circ} \mathrm{C}\right)(=P \ddot{A})$ zu $53,5 \mathrm{~g}(0,6 \mathrm{Mol})$ 2-Dimethylaminoäthanol in $200 \mathrm{ml} P \ddot{A}$. Ausb. 27,3-29 $\mathrm{g}(75-80 \%)$.

b) Gemäß Rk. (8) aus $27,7 \mathrm{~g}(0,1 \mathrm{Mol})$ III und 17,8 $\mathrm{g}$ (0,2 Mol) 2-Dimethylaminoäthanol in $35 \mathrm{ml} P \ddot{A}$ nach Zugabe katalytischer Mengen an Butyllithium. Ausb. 34,6 g (95\%).

$N, N$-Diäthyl-2-(p-bromphenyl-methyl-phenylsilyloxy)-äthylamin (B 2): Gemäß Rk. (3) in Analogie zur Darstellung von A 2 aus $31,2 \mathrm{~g}(0,1 \mathrm{Mol}) \mathrm{I}$ und 70,3 $\mathrm{g}(0,6 \mathrm{Mol})$ 2-Diäthylaminoäthanol. Ausb. 29,4-31,4 $\mathrm{g}(75-80 \%)$.

$N$-Morpholino-2-(p-bromphenyl-methyl-phenylsilyloxy)-äthylamin (G 2): Gomäß Rk. (3) in Analogie zur Darstellung von A 2 aus $31,2 \mathrm{~g}(0,1 \mathrm{Mol}) \mathrm{I}$ und 78,7 $\mathrm{g}(0,6 \mathrm{Mol}) 2$-Morpholinoäthanol. An Stelle von $P \ddot{A}$ wurde Benzol als Lösungsnaittel verwandt. Ausb. 30,5-32,5 g (75-80\%).

3. Chemische und physikalische Eigenschaften des SilaMebrophenhydramins, seiner Vorstufen und Homologen

I-III sind wasserklare, ihrer SiCl- bzw. SiH-Gruppierungen wegen hydrolyseempfindliche Flüssigkeiten, die - mit Ausnahme von III an der Luft rauchen. Sie lösen sich gut in den üblichen inerten organischen Lösungsmitteln und sind unter vermindertem Druck unzersetzt destillierbar (Siedepunkte siehe Tab. 1).

I und III wurden bereits in der Literatur ${ }^{6,7}$ beschrieben. Die von uns ermittelten Siedepunkte, Brechungsindices und Dichten stimmen weitgehend mit den Literaturangaben überein. 
Tabelle 1. Physikalische Daten und Ausbeuten der Verbindungen I-III sowie A 2-C 2

\begin{tabular}{|c|c|c|c|c|c|c|c|c|c|c|}
\hline Lfd. & Sdp. & & & & & & & $\varepsilon_{E}^{\mathrm{a}}$ & Aus & isboute \\
\hline Nr. & & 'Torr & $D_{4}^{20}$ & $n_{\mathrm{D}}^{20}$ & ber. & gef. & ber. & gef. & Rk. & $\%$ \\
\hline I & $128^{b}$ & 0,5 & & $1,5978 \mathrm{~b}$ & 77,50 & 77,39 & 492,9 & 498,0 & 2 & 29 \\
\hline II & 100 & 20 & & 1,5 & 53,1 & & 366,2 & 367,4 & $\tilde{5}$ & 26 \\
\hline III & $89^{c}$ & 0,01 & & & 73,56 & 73,62 & 441,0 & 442,5 & $\begin{array}{l}7 \\
6\end{array}$ & $\begin{array}{l}85 \\
29\end{array}$ \\
\hline 1 & $135-140$ & $0,3^{c}$ & & & & & & & & \\
\hline A 2 & $126-127$ & 0,01 & 1,2171 & 1,5590 & 96,36 & 96,66 & 566,5 & 568,1 & $\begin{array}{l}3 \\
8\end{array}$ & $\begin{array}{c}75-80 \\
95\end{array}$ \\
\hline 1 & $150-155$ & $0,5^{d}$ & & & & & & & & \\
\hline 2 & $141-143$ & 0,01 & & & 105,6 & & 607,7 & & 37 & $75-8$ \\
\hline 22 & $179-180$ & 0,01 & 1,2628 & 1,5685 & 105,3 & 105,4 & 635,4 & 637,4 & 37 & $75-8$ \\
\hline
\end{tabular}

a Für die $\mathrm{C}-\mathrm{Br}$-Bindung (C aromatisch) wurden die entsprechenden Bindungsinkremente aus den physikalischen Daten folgender Verbindungen berechnet: Brombenzol, p-Bromtoluol, m-Bromtoluol, o-Bromtoluol, 1 Brom-4-isopropylbenzol. Zur Berechnung der erforderlichen $n_{\mathrm{D}}^{20}$ - und $D_{4}^{20}$-Werte dienten Literaturwerte (Handbook of Chemistry and Physics, The Chemical Rubber Co., Ohio 1971). Berechnete Mittelwerte: $M R_{E}$ $(\mathrm{C}-\mathrm{Br})=130,23 ; M R_{L}(\mathrm{C}-\mathrm{Br})=9,45$.

b Literaturwerte ${ }^{6}: \operatorname{Sdp}_{1} 130-131^{\circ} \mathrm{C}, D_{20} 1,3575, n_{\mathrm{D}}^{20} 1,5950$.

c Literaturwerte ${ }^{\mathrm{r}}$ : Sdp. $138-140^{\circ} \mathrm{C}, D_{20} 1,2841, n_{\mathrm{D}}^{20} 1,5970$.

d L. Novák und M. Protiva, Chem. Abstr. 54, 8766 (1960).

Tabelle 2. Elementaranalytische Daten der Verbindung II sowie A 2-G 2

\begin{tabular}{|c|c|c|c|c|c|c|c|}
\hline $\begin{array}{l}\text { Lfd. } \\
\text { Nr. }\end{array}$ & Summenformel & $\begin{array}{l}\text { Molgew. } \\
\text { ber. }{ }^{a}\end{array}$ & $\begin{array}{l}\% \mathrm{C} \\
\text { ber. } \\
\text { gef. }\end{array}$ & $\begin{array}{c}\% \mathrm{H} \\
\text { ber. } \\
\text { gef. }\end{array}$ & $\begin{array}{l}\% \mathrm{Cl} \\
\text { ber. } \\
\text { gef. }\end{array}$ & $\begin{array}{l}\% \mathrm{Br} \\
\text { ber. } \\
\text { gef. }\end{array}$ & $\begin{array}{l}\% \mathrm{~N} \\
\text { ber. } \\
\text { gef. }\end{array}$ \\
\hline II & $\mathrm{C}_{7} \mathrm{H}_{8} \mathrm{BrClSi}$ & 235,59 & $\begin{array}{l}35,69 \\
36,77\end{array}$ & $\begin{array}{l}3,42 \\
3,64\end{array}$ & $\begin{array}{l}15,05 \\
14,95\end{array}$ & $\begin{array}{l}33,92 \\
33,74\end{array}$ & \\
\hline & & & & & $\% \mathrm{Si}$ & & \\
\hline A 2 & $\mathrm{C}_{17} \mathrm{H}_{22} \mathrm{BrNOSi}$ & 364,37 & $\begin{array}{l}56,04 \\
\tilde{5} 5,90\end{array}$ & $\begin{array}{l}6,09 \\
6,10\end{array}$ & $\begin{array}{l}7,71 \\
7,89\end{array}$ & $\begin{array}{l}21,93 \\
22,15\end{array}$ & $\begin{array}{l}3,84 \\
3,92\end{array}$ \\
\hline B 2 & $\mathrm{C}_{19} \mathrm{H}_{26} \mathrm{BrNOSi}$ & 392,42 & $\begin{array}{l}58,15 \\
57,95\end{array}$ & $\begin{array}{l}6,68 \\
6,59\end{array}$ & $\begin{array}{l}7,16 \\
6,83\end{array}$ & $\begin{array}{l}20,36 \\
20,40\end{array}$ & $\begin{array}{l}3,57 \\
3,85\end{array}$ \\
\hline C 2 & $\mathrm{C}_{19} \mathrm{H}_{24} \mathrm{BrNO}_{2} \mathrm{Si}$ & 406,40 & $\begin{array}{l}56,15 \\
55,15\end{array}$ & $\begin{array}{l}5,95 \\
5,72\end{array}$ & $\begin{array}{l}6,91 \\
6,55\end{array}$ & $\begin{array}{l}19,66 \\
19,48\end{array}$ & $\begin{array}{l}3,45 \\
3,20\end{array}$ \\
\hline
\end{tabular}

a Bestätigt durch Molpeak im Massenspektrum (Tab. 4). 
Das Sila-Mebrophenhydramin (A 2) sowie die Homologen $B 2$ und $\mathbf{C} 2$ sind wasserklare, ölige Flüssigkeiten, die sich unzersetzt im Vak. destillieren lassen. Sie sind in den gängigen organischen Solventien leicht löslich. Die Löslichkeit in Wasser ist dagegen sehr schlecht. Es kommt zur Ausbildung von Zweiphasensystemen, an deren Grenzflächen Hydrolysereaktionen ablaufen: Durch Spaltung der Si-OCGruppierungen entstehen das entsprechende Silanol und die dazuge-

Tabelle 3. 1H-NMR-Spektren der Verbindungen $I-I I l$ sowie A 2-C 2. Chemische Verschiebung in $\delta[\mathrm{ppm}]$, relative Intensitäten in Klammern, S Singulett, D Dublett, T Triplett, Q Quartett, M Multiplett. Lösungs. mittel: $\mathrm{CCl}_{4}$ (I-III) bzw. $\mathrm{CDCl}_{3}$ (A 2-G 2), Lock extern: $\mathrm{H}_{2} \mathrm{O}$, Standard intern: $T M S$

\begin{tabular}{|c|c|c|c|c|c|c|c|}
\hline $\begin{array}{l}\text { Lfd. } \\
\text { Nr. }\end{array}$ & $\begin{array}{l}\text { aromatische } \\
\text { Protonen }\end{array}$ & $\mathrm{SiH}$ & $\mathrm{OCH}_{2} \mathrm{C}$ & $\mathrm{NCH}_{2} \mathrm{C}$ & $\mathrm{NCH}_{3}$ & $\mathrm{CCH}_{3}$ & $\mathrm{SiCH}_{3}$ \\
\hline $\mathrm{I}$ & $7,8-7,1 \mathrm{M}(9)$ & & & & & & $0,84 \mathrm{~S}(3)$ \\
\hline II & $7,50 M(4)^{\mathrm{f}}$ & $5,27 \mathrm{Q}(1)^{\mathrm{a}}$ & & & & & $0,64 \mathrm{D}(3)^{\mathrm{a}}$ \\
\hline III & $7,7-6,9 \mathrm{M}(9)$ & $4,89 Q(1)^{b}$ & & & & & $0,54 \mathrm{D}(3)^{\mathrm{b}}$ \\
\hline A 2 & $7,9-7,0 \mathrm{M}(9)$ & & $3,76 \mathrm{~T}(2) \mathrm{c}$ & $2,43 \mathrm{~T}(2) \mathrm{c}$ & $2,12 \mathrm{~S}(6)$ & & $0,61 \mathrm{~S}(3)$ \\
\hline B 2 & $7,9-7,0 \mathrm{M}(9)$ & & $3,75 \mathrm{~T}(2)^{\mathrm{d}}$ & $\begin{array}{l}2,57 \mathrm{~T}(2)^{\mathrm{d}} \\
2,48 \mathrm{Q}(4)^{\mathrm{e}}\end{array}$ & & $0,92 \mathrm{~T}(6)^{\mathrm{e}}$ & $0,60 \mathrm{~S}(3)$ \\
\hline C 2 & $7,8-7,0 \mathrm{M}(9)$ & & $3,4-4,0 \mathrm{M}(6)$ & $2,2-2,7 \mathrm{M}(6)$ & & & $0,62 \mathrm{~S}(3)$ \\
\hline
\end{tabular}

Kopplungskonstanten $J_{\text {vic }} \mathrm{H}-\mathrm{Si}-\mathrm{C}-\mathrm{H}$. a 3,3 . b 3,$8 ; \mathrm{H}-\mathrm{C}-\mathrm{C}-\mathrm{H}$. c 6,0. d 6,5. e 7,0, alle $\pm 0,2 \mathrm{~Hz}$. ${ }^{\mathrm{f}}$ Zentrum eines symmetrischen Multipletts vom $A A^{\prime} B B^{\prime}$-System.

hörigen Aminoalkohole. Bei einer guten Verteilung und einer kleinen Konzentration (10-6 Mol/l) der Verbindungen in Tyrode-Lösung (Was. ser, gepuffert auf pH 7,4; Temp. $37^{\circ} \mathrm{C}$ ) erfolgt der hydrolytische $\mathrm{Ab}$ bau relativ rasch, wie sich an Hand pharmakologischer Messungen erkennen ließ (vgl. Kap. 5).

4. Die Strukturermittlung des Sila-Mebrophenhydramins und seiner Homologen

sowie ihrer Vorstufen erfolgte wie bei den zuvor beschriebenen basischen Sila-Benzhydryläthern ${ }^{1,} 4$ neben den Elementaranalysen (Tab. 2) hauptsächlich über die Protonenresonanz- und Massenspektren. Die entsprechenden spektroskopischen Daten von I-III sowie A 2-G 2 sind in Tab. 3 und 4 aufgeführt. Für eine nähere Beschreibung der Spek- 
Tabelle 4. m/e-Werte der Massenspektren der Verbindungen I-III sowie A 2 bis C 2. In runden Klammern relative Intensitäten (Basispeak $=100$ ), ferner Halogenisotopenmuster sowie Zuordnung der Peaks zu charakteristischen Schlüsselbruchstücken. Probeneinführung durch Direkteinlaß (A 2-C 2) oder Hochtemperatureinlaß (I, II, III), Elektronenstoßionisierung mit $70 \mathrm{eV}$

\begin{tabular}{|c|c|c|c|c|}
\hline $\begin{array}{l}\text { Lfd. } \\
\text { Nr. }\end{array}$ & $m / e$ & $\begin{array}{l}\text { relative } \\
\text { Intensität }\end{array}$ & $\begin{array}{l}\text { Halogeniso- } \\
\text { topenmuster }\end{array}$ & Fragment \\
\hline$I$ & $\begin{array}{l}310 / 312 / 314 \\
295 / 297 / 299 \\
77 \\
63 / 65\end{array}$ & $\begin{array}{l}(26 / 35 / 11) \\
(75 / 100 / 30) \\
(5) \\
(20 / 8)\end{array}$ & $\begin{array}{l}\mathrm{BrCl} \\
\mathrm{BrCl} \\
\mathrm{Cl}\end{array}$ & $\begin{array}{l}M^{+} \\
M^{+}-\mathrm{CH}_{3} \\
\mathrm{C}_{6} \mathrm{H}_{5}^{+} \\
\mathrm{SiCl}^{+}\end{array}$ \\
\hline II & $\begin{array}{l}234 / 236 / 238 \\
233 / 235 / 237 \\
219 / 221 / 223 \\
183 / 185 \\
77 \\
63 / 65 \\
43\end{array}$ & $\begin{array}{l}(28 / 37 / 11) \\
(14 / 22 / 11) \\
(82 / 100 / 28) \\
(3 / 3) \\
(22) \\
(72 / 27) \\
(99)\end{array}$ & $\begin{array}{l}\mathrm{BrCl} \\
\mathrm{BrCl} \\
\mathrm{BrCl} \\
\mathrm{Br}\end{array}$ & $\begin{array}{l}M^{+} \\
M^{+}-\mathrm{H} \\
M^{+}-\mathrm{CH}_{3} \\
\mathrm{SiC}_{6} \mathrm{H}_{4} \mathrm{Br}^{+} \\
\mathrm{C}_{6} \mathbf{H}_{5}{ }^{+} \\
\mathrm{SiCl}^{+} \\
\mathrm{SiCH}_{3}+\end{array}$ \\
\hline III & $\begin{array}{l}276 / 278 \\
275 / 277 \\
261 / 263 \\
120 \\
183 / 185 \\
105 \\
\quad 43\end{array}$ & $\begin{array}{l}(29 / 28) \\
(14 / 19) \\
(99 / 100) \\
(96) \\
(33 / 29) \\
(90) \\
(31)\end{array}$ & $\begin{array}{l}\mathrm{Br} \\
\mathrm{Br} \\
\mathrm{Br}\end{array}$ & $\begin{array}{l}M^{+} \\
M^{+}-\mathrm{H} \\
M^{+}-\mathrm{CH}_{3} \\
M^{+}-\left(\mathrm{C}_{6} \mathrm{H}_{4} \mathrm{Br}+\mathrm{H}\right) \\
\mathrm{SiC}_{6} \mathrm{H}_{4} \mathrm{Br}^{+} \\
\mathrm{SiC}_{6} \mathrm{H}_{5}^{+} \\
\mathrm{SiCH}_{3}^{+}\end{array}$ \\
\hline A 2 & $\begin{array}{l}363 / 365 \\
348 / 350 \\
277 / 279 \\
275 / 277 \\
105 \\
77 \\
58\end{array}$ & $\begin{array}{c}(41 / 44) \\
(7 / 7) \\
(46 / 16) \\
(32 / 46) \\
(20) \\
(6) \\
(100)\end{array}$ & $\begin{array}{l}\mathrm{Br} \\
\mathrm{Br} \\
\mathrm{Br} \\
\mathrm{Br}\end{array}$ & $\begin{array}{l}M^{+} \\
M^{+}-\mathrm{CH}_{3} \\
\mathrm{Si}\left(\mathrm{BrC}_{6} \mathrm{H}_{4}\right)\left(\mathrm{C}_{6} \mathrm{H}_{5}\right) \mathrm{OH}^{+} \\
M^{+}-\mathrm{OCH}_{2} \mathrm{CH}_{2} \mathrm{~N}\left(\mathrm{CH}_{3}\right)_{2} \\
\mathrm{SiC}_{6} \mathrm{H}_{5} \\
\mathrm{C}_{6} \mathrm{H}_{5}^{+} \\
\mathrm{CH}_{2}=\mathrm{N}\left(\mathrm{CH}_{3}\right)_{2}^{+}\end{array}$ \\
\hline B 2 & $\begin{array}{l}391 / 393 \\
376 / 378 \\
277 / 279 \\
275 / 277 \\
105 \\
77 \\
86 \\
58\end{array}$ & $\begin{array}{c}(9 / 10) \\
(1 / 1) \\
(7 / 1) \\
(4 / 7) \\
(3) \\
(1) \\
(100) \\
(32)\end{array}$ & $\begin{array}{l}\mathrm{Br} \\
\mathrm{Br} \\
\mathrm{Br} \\
\mathrm{Br}\end{array}$ & $\begin{array}{l}M^{+} \\
M^{+}-\mathrm{CH}_{3} \\
\text { wie bei A } 2 \\
\text { wie bei A } 2 \\
\text { wie bei A } 2 \\
\text { wie bei A } 2 \\
\mathrm{CH}_{2}=\mathrm{N}_{2}\left(\mathrm{C}_{2} \mathrm{H}_{5}\right)_{2}^{+} \\
\mathrm{CH}_{2}=\mathrm{NH}\left(\mathrm{C}_{2} \mathrm{H}_{5}\right)^{+}\end{array}$ \\
\hline C 2 & $\begin{array}{l}405 / 407 \\
390 / 392 \\
277 / 279 \\
275 / 277 \\
105 \\
77\end{array}$ & $\begin{array}{c}(75 / 78) \\
(2 / 2) \\
(62 / 26) \\
(41 / 62) \\
(27) \\
(8)\end{array}$ & $\begin{array}{l}\mathrm{Br} \\
\mathrm{Br} \\
\mathrm{Br} \\
\mathrm{Br}\end{array}$ & $\begin{array}{l}M^{+} \\
M^{+}-\mathrm{CH}_{3} \\
\text { wie bei A } 2 \\
\text { wie bei A } 2 \\
\text { wie bei A } 2 \\
\text { wie bei A } 2\end{array}$ \\
\hline & 100 & $(100)$ & & $\mathrm{CH}_{2}=\mathrm{N} \bigcirc \mathrm{O}^{+}$ \\
\hline
\end{tabular}


tren sei auf die früheren Mitteilungen ${ }^{1,}{ }^{4}$ verwiesen, in denen die Spektren analoger Verbindungen etwas ausführlicher erläutert werden. Besonders erwāhnenswert ist die gute Übersichtlichkeit und Deutbarkeit der Mas. senspektren auf Grund der Halogen-Isotopenmuster.

Die Infrarotspektren sind schwierig zuzuordnen (tabellarische Aufzählung vgl. ${ }^{2}$ ). Strukturbeweisend für II und III ist die v SiH-Schwingung bei 2185 bzw. $2125 \mathrm{~cm}^{-1}$, für alle die Anwesenheit der $\delta_{\mathrm{s}}-\mathrm{SiCH}_{3}-\mathrm{Sch}$ wingung bei $1250-1260 \mathrm{~cm}^{-1}$.

5. Pharmakologische Eigenschaften der Sila-Pharmaka A 2 und $\mathbf{C} 2$

Pharmakologische Untersuchungen - sie wurden in enger Zusammenarbeit am Institut für Pharmakologie und Toxilsologie der Technischen Universität Braunschweig von H.-U. Rossée und $F$. Meyer durchgeführt; für ausführliche Informationen sei auf die Originalarbeit Rossée/Meyer verwiesen ${ }^{8}$ - der Sila-Pharmaka A 2 und C 2 am isolierten Meerschweinchen-Ileum ergaben, daß beide Verbindungen pharmakodynamisch als kompetitive Antagonisten der Spasmodika Histamin und Carbachol wirksam sind, wobei der Histamin-Antagonismus am stärksten ausgeprägt ist. Die Verbindungen erfüllen damit - ebenso wie die schon früher von uns beschriebenen Sila-Antihistaminika - die von der Struktur her an sie gestellten Erwartungen.

Das Sila-Mebrophenhydramin ist sowohl histaminolytisch als auch cholinolytisch schwächer wirksam als das Sila-Mephenhydramin und Sila-Chlorphenoxamin. In diesem Zusammenhang überrascht, daß der Antihistamin-Effekt des Sila-Mebrophenhydramins deutlich schwächer ist als der des Sila-Mephenhydramins, während für die beiden analogen C-Muttersubstanzen eine genau entgegengesetzte Struktur-WirkungsBeziehung beschrieben wird ${ }^{3}$.

Der direkte Vergleich zwischen A 2 und G 2 zeigt, daß die Dimethyl. amino-Verbindung bezüglich beider Wirkkomponenten die aktivere ist. Analoge Strulktur-Wirkungs-Beziehungen fanden Arnold et al. ${ }^{9}$ beim Vergleich des Diphenhydramins (Benzhydryl-dimethylaminoäthyläther) mit der strukturanalogen Morpholino-Verbindung.

Bezüglich ihrer Wirkdauer unterscheiden sich A 2 und C 2 ebenfalls - wie die schon früher von uns beschriebenen Sila-Pharmaka ganz erheblich von ihren C-Muttersubstanzen. Infolge der Hydrolyselabilität der Si-OC-Gruppierung nimmt die Wirkintensität mit der Zeit schnell ab. Die von Rossée 8 auf biologischem Wege gemessenen Hydrolysezeiten (Konzentration der Sila-Pharmaka: $10^{-6} \mathrm{Mol} / \mathrm{l}$ ) in Tyrode-Lösung betragen für A 214 Min. und für C 2 etwa 20 Min. 
Unser Dank gilt dem Verband der Chemischen Industrie, Frankfurt am Main, für die Unterstützung mit Sachmitteln, der Bayer AG, Leverkusen, für die Überlassung von Chlorsilanen, sowie Prof. Dr. $F$. Meyer und Dr. H..U. Rossée für viele wertvolle Hinweise und Diskussionen. Ebenso danken wir Herrn Dr. H.M. Schiebel für die Hilfestellung bei der Messung und Auswertung der Massenspektren.

\section{Literatur}

1 2. Mitt.: R. Tacke und U. Wannagat, Mh. Chem. 107, 111 (1976)

2 Mit Auszügen aus der Dissertation R. Tacke, Techn. Univ. Braunschweig 1974.

3 Z. Votava, J. Metyšová und Z. Horáková, Arzneimittel-Forsch. 11, 531 (1961).

4 R. Tacke und $U$. Wannagat, Mh. Chem. 106, 1005 (1975).

${ }^{5}$ L. Novále und M. Protiva, Collect. Czech. Chem. Commun. 24, 3966 (1959).

6 K. A. Andrianov, V.E. Nikitenkov und N. N. Sokolov, Izvest. Akad. Nauk SSSR, Otdel. Khim. Nauk 1960, 1224 ; Chem. Abstr. 55, 429 (1961).

7 N. S. Nametkin, N. A. Pritula, A. V. Topchiev und T. I. Chernysheva, Neftekhimiya 2, 632 (1962); Chem. Abstr. 58, 11392 (1963).

${ }^{8} H .-U$. Rossée und F. Meyer, unveröffentlicht, H.-U. Rossée, Dissertation Technische Universität Braunschweig 1974.

9 H. Arnold, N. Brock, E. Kühas und D. Lorenz, Arzneimittel-Forsch. 4, 189 (1954).

Korrespondenz und Sonderdrucke:

Dr. R. Tacke

Institut für Anorganische Chemie

Technische Universität Braunschweig

Pockelsstraße 4

D-3300 Braunschweig

Bundesrepublik Deutschland 